\title{
A Comparison of 2-CUSUM Stopping Rules for Quickest Detection of Two-sided Alternatives in a Brownian Motion Model
}

\author{
O. Hadjiliadis \\ Department of Mathematics, Brooklyn College, CUNY, Brooklyn, NY, USA \\ G. Hernandez del-Valle \\ Department of Statistics, Columbia University, New York, NY, USA \\ I. Stamos \\ Department of Computer Science, Hunter College, CUNY, New York, NY, USA
}

\begin{abstract}
This work compares the performance of all existing 2-CUSUM stopping rules used in the problem of sequential detection of a change in the drift of a Brownian motion in the case of two-sided alternatives. As a performance measure an extended Lorden criterion is used. According to this criterion the optimal stopping rule is an equalizer rule. This paper compares the performance of the modified drift harmonic mean 2-CUSUM equalizer rules with the performance of the best classical 2-CUSUM equalizer rule whose threshold parameters are chosen so that equalization is achieved. This comparison is made possible through the derivation of a closed-form formula for the expected value of a general classical 2-CUSUM stopping rule.
\end{abstract}

Keywords: 2-CUSUM; Change-point detection; Two-sided detection; Sequential detection; Brownian motion.

Subject Classifications: 62L15; 60G40; 62F12.

\section{INTRODUCTION}

The need for statistical surveillance has been noted in many different areas (see, e.g., Anderson et al., 2004; Basseville and Nikiforov, 1993; Doerschuk et al., 1986; Willsky et al., 1980). This area can be studied mathematically by considering the problem of detecting a change in a stochastic process through sequential observations. In this formalism, we seek a stopping rule $\tau$ that detects a change point $\theta$ while at the same time controls the mean time to false alarms. In other words, at each decision time point, $t$, we want to discriminate between the two states of the process: the state, $\{t<\theta\}$, and the state $\{t \geq \theta\}$. More specifically, the stopping rule $\tau$ minimizes the detection delay of the change under the constraint on the mean time to false alarms.

A useful model for studying such problems is a Brownian motion whose drift changes from one constant to another at the unknown change point. In particular, for the case of

Address correspondence to O. Hadjiliadis, Department of Mathematics, 1314N Ingersoll Hall, Brooklyn College of the City University of New York, Brooklyn, NY, 11209, USA; E-mail:

ohadjiliadis@brooklyn.cuny.edu 
one-sided alternatives, in which the change in the drift is a known constant, the traditional Page's CUSUM is known to be optimal for any fixed value of the mean time to false alarms (see Beibel, 1996; Shiryaev, 1996).

The problem of detecting a change in the drift of a Brownian motion with two-sided alternatives is considerably more difficult than that with one-sided alternatives. This paper is a continuation of the work started in Hadjiliadis (2005), Hadjiliadis and Moustakides (2006), and Hadjiliadis and Poor (2008). In Hadjiliadis and Moustakides (2006) it is conjectured but not proven that within the class of 2-CUSUM harmonic mean rules, drift equalizer rules are best and two strong asymptotic optimality results as the mean time to false alarms tends to infinity are presented both in the symmetric and the non-symmetric case. These asymptotic results enhance the 2-CUSUM asymptotic optimality results of Tartakovsky (1994). In Hadjiliadis (2005), it is seen that within the class of modified drift 2-CUSUM harmonic mean rules, the best rules are those for which the drift parameters of the modified drift 2-CUSUM harmonic mean rules, $\lambda_{1}$ and $\lambda_{2}$, are chosen so that $\lambda_{2}-\lambda_{1}=2\left(\mu_{2}-\mu_{1}\right)$, for any value of the mean time to false alarms. (Here, $\mu_{1}$ and $-\mu_{2}$ are the possible drift parameters assumed after the change). In Hadjiliadis and Poor (2008) it is proven that the optimal solution to the problem of quickest detection of two-sided alternatives has to be an equalizer rule. In the same paper it is proven that the best amongst the classical 2-CUSUM stopping rules is unique and is a harmonic mean rule in the case of a symmetric change in the drift while it is a non-harmonic mean rule with threshold parameters $\nu_{1}>\nu_{2}\left(\nu_{1}<\nu_{2}\right)$ when $\mu_{1}>\mu_{2}\left(\mu_{1}<\mu_{2}\right)$ for any value of the mean time to false alarms. All existing results are summarized in the following table

Table 1. Existing results regarding 2-CUSUM stopping rules

\begin{tabular}{|l|l|l|}
\hline & Classical & Modified drift \\
\hline Harmonic mean rules & $\begin{array}{l}\text { Best amongst all classical rules } \\
\text { in the symmetric case }\end{array}$ & $\begin{array}{l}\text { Best for } \\
\lambda_{2}-\lambda_{1}=2\left(\mu_{2}-\mu_{1}\right)\end{array}$ \\
\hline Non-harmonic mean rules & $\begin{array}{l}\text { Best amongst all classical rules } \\
\text { in the non-symmetric case }\end{array}$ & \\
\hline
\end{tabular}

In this paper, we begin by deriving a closed-form formula for the first moment of a general 2-CUSUM stopping rule based on the Brownian motion model using renewal arguments and Anderson (1960). This rule, although similar, is different from the ones treated in Khan (2007) and Yashchin (1985), which are based on the drawdown and upward rally processes also studied in Hadjiliadis and Vecer (2006). The fact that the optimal stopping rule is an equalizer rule (see Hadjiliadis and Poor, 2008) gives rise to the natural question of comparing the best classical 2-CUSUM equalizer rule with the modified drift 2-CUSUM harmonic mean equalizer rule. In this paper, we compare the detection delay of the former with the latter both in the symmetric and the non-symmetric case for a given fixed level of the mean time to false alarms. In the symmetric case, it is seen that the modified drift 2-CUSUM harmonic mean rule displays a slightly better performance than the classical 2-CUSUM harmonic mean equalizer rule, which manifests itself for small values 
of a two-sided change in the drift parameter and of the mean time to false alarms. In the non-symmetric case the results involved are very interesting and to some extent surprising. The first observation is that although for the modified drift parameter 2-CUSUM harmonic mean equalizer rules the relationship that the modified drifts have to satisfy for equalization is linear (see Hadjiliadis, 2005), the relationship that the threshold parameters $\nu_{1}$ and $\nu_{2}$ have to satisfy for equalization in the classical 2-CUSUM stopping rule is much more involved. Moreover, it is seen that the classical 2-CUSUM equalizer rule outperforms the modified drift parameter 2-CUSUM harmonic mean equalizer rule for effectively all values of the mean time to false alarms. Interestingly, the performance of both types of rules as the mean time to false alarms tends to infinity is identical. Further details will be presented in the main body of the paper.

In Section 2, we mathematically formulate the problem of change-point detection with two-sided alternatives in a Brownian motion model. In Section 3, we derive an explicit formula for the first moment of a general 2-CUSUM stopping rule under all relevant measures. In Section 4, we present the comparisons of the modified drift 2-CUSUM harmonic mean equalizer stopping rule to the classical 2-CUSUM harmonic mean rule in the symmetric case. In Section 5, we concentrate on the non-symmetric case. We first provide a qualitative analysis of the relationship between the threshold parameters of the classical 2CUSUM equalizer rules and then proceed to compare its performance to the modified drift 2-CUSUM harmonic mean equalizer rules. Finally, in Section 6, we conclude with some closing remarks. Appendices A and B contain the Mathematica code used to perform the above comparisons.

\section{MATHEMATICAL FORMULATION}

We sequentially observe a process $\left\{\xi_{t}\right\}$ with the following dynamics:

$$
d \xi_{t}=\left\{\begin{array}{cc}
d w_{t} & t \leq \theta \\
& \\
\mu_{1} d t+d w_{t} & \\
\text { or } & t \geq \theta \\
-\mu_{2} d t+d w_{t} &
\end{array}\right.
$$

where $\theta$, the time of change, is assumed to be deterministic but unknown; $\left\{w_{t}\right\}$ is a standard Brownian motion process; $\mu_{i}$, the possible drifts to which the process can change, are assumed to be known, but the specific drift to which the process is changing is unknown. Both $\mu_{1}$ and $\mu_{2}$ are assumed to be positive.

The probability triplet consists of $\left(C[0, \infty], \cup_{t>0} \mathcal{F}_{t}\right)$, where $\mathcal{F}_{t}=\sigma\left\{\xi_{s}, 0<s \leq t\right\}$ and the families of probability measures $\left\{\mathcal{P}_{\theta}^{i}\right\}, \theta \in[0, \infty)$, whenever the change is $\mu_{i}, i=1,2$, and $\mathcal{P}_{\infty}$ (the Wiener measure).

Our goal is to detect a change by means of a stopping rule $\tau$ adapted to the filtration $\mathcal{F}_{t}$. As a performance measure for this stopping rule we propose an extended Lorden criterion 
(see Hadjiliadis and Moustakides, 2006)

$$
J_{L}(\tau)=\max \left\{J_{1}(\tau), J_{2}(\tau)\right\}
$$

where $J_{i}(\tau)=\sup _{\theta} \operatorname{essup} E_{\theta}^{i}\left[(\tau-\theta)^{+} \mid \mathcal{F}_{\theta}\right], i=1,2$. This gives rise to the following min-max constrained optimization problem:

$$
\begin{array}{r}
\inf _{\tau} J_{L}(\tau) \\
\text { subject to } E_{\infty}[\tau] \geq \gamma,
\end{array}
$$

where the constraint specifies the minimum allowable mean time to false alarms. As discussed in Moustakides (1986), in seeking solutions to the above problem, we can restrict our attention to stopping times that achieve the false alarm constraint with equality; that is, stopping rules $\tau$ for which

$$
E_{\infty}[\tau]=\gamma
$$

As argued in Hadjiliadis and Poor (2008) the optimal stopping rule for problem (2.2) has to satisfy

$$
J_{1}(\tau)=J_{2}(\tau) .
$$

We now proceed to define the normalized CUSUM processes and their corresponding one-sided CUSUM stopping rules.

Definition 2.1 Let $\nu_{1}>0$ and $\nu_{2}>0$. Define

$$
\begin{array}{ll}
\text { 1. } & u_{t}^{+}=\frac{\log \frac{d P_{0}^{1}}{d P_{\infty}} \mid \mathcal{F}_{t}}{\mu_{1}}=\xi_{t}-\frac{1}{2} \mu_{1} t ; m_{t}^{+}=\inf _{s \leq t} u_{s}^{+} ; y_{t}^{+}=u_{t}^{+}-m_{t}^{+} ; \tau_{1}\left(\nu_{1}\right)=\inf \{t> \\
& \left.0 ; y_{t}^{+} \geq \nu_{1}\right\}, \\
\text { 2. } & u_{t}^{-}=\frac{\log \frac{d P_{0}^{2}}{d P_{\infty}} \mid \mathcal{F}_{t}}{\mu_{2}}=-\xi_{t}-\frac{1}{2} \mu_{2} t ; m_{t}^{-}=\inf _{s \leq t} u_{s}^{-} ; y_{t}^{-}=u_{t}^{-}-m_{t}^{-} ; \tau_{2}\left(\nu_{2}\right)=\inf \{t> \\
& \left.0 ; y_{t}^{-} \geq \nu_{2}\right\} .
\end{array}
$$

That is, $\tau_{1}\left(\nu_{1}\right)$ and $\tau_{2}\left(\nu_{2}\right)$ are the first times that the processes $y_{t}^{+}$and $y_{t}^{-}$reach their corresponding thresholds $\nu_{1}$ and $\nu_{2}$ respectively. The classical 2-CUSUM stopping rules are then of the form $\tau\left(\nu_{1}, \nu_{2}\right)=\tau_{1}\left(\nu_{1}\right) \wedge \tau_{2}\left(\nu_{2}\right)$.

We classify 2-CUSUM rules according to the class $\mathcal{G}=\left\{\tau\left(\nu_{1}, \nu_{2}\right) ; \nu_{1}=\nu_{2}\right\}$ of harmonic mean rules and the classes $\mathcal{C}_{i}=\left\{\tau\left(\nu_{i}, \nu_{j}\right) \mid \nu_{i}>\nu_{j}>0, i \neq j\right\}$ of non-harmonic mean rules.

Remark 2.1 It is useful at this stage to contrast the stopping time $\tau\left(\nu_{1}, \nu_{2}\right)$ to the one used in Khan (2007). The one considered in Khan (2007) using our notation, is defined as $T=T_{1}\left(h_{1}\right) \wedge T_{2}\left(h_{2}\right)$, where $T_{1}\left(h_{1}\right)$ is the first time that the process $Y_{t}^{+}=\xi_{t}-\inf _{s \leq t} \xi_{s}$ reaches the threshold $h_{1}$ and $T_{2}\left(h_{2}\right)$ is the first time that the process $Y_{t}^{-}=\sup _{s \leq t} \xi_{s}-\xi_{t}$ reaches the threshold $h_{2}$. It is therefore a different stopping rule. We will revisit this point in Remark 3.1.

\footnotetext{
${ }^{1}$ Notice that log throughout the paper denotes the logarithm with base $e$.
} 
We now proceed to define the modified drift 2-CUSUM stopping rules as follows.

Definition 2.2 Let $\lambda_{1}>0, \lambda_{2}>0, \nu_{1}>0$ and $\nu_{2}>0$. Define

1. $u_{t}^{+}\left(\lambda_{1}\right)=\xi_{t}-\frac{1}{2} \lambda_{1} t ; m_{t}^{+}\left(\lambda_{1}\right)=\inf _{s \leq t} u_{s}^{+}\left(\lambda_{1}\right) ; y_{t}^{+}\left(\lambda_{1}\right)=u_{t}^{+}\left(\lambda_{1}\right)-m_{t}^{+}\left(\lambda_{1}\right)$; $\tau_{1}\left(\lambda_{1}, \nu_{1}\right)=\inf \left\{t>0 ; y_{t}^{+}\left(\lambda_{1}\right) \geq \nu_{1}\right\}$,

2. $u_{t}^{-}\left(\lambda_{2}\right)=-\xi_{t}-\frac{1}{2} \lambda_{2} t ; m_{t}^{-}\left(\lambda_{2}\right)=\inf _{s \leq t} u_{s}^{-}\left(\lambda_{2}\right) ; y_{t}^{-}\left(\lambda_{2}\right)=u_{t}^{-}\left(\lambda_{2}\right)-m_{t}^{-}\left(\lambda_{2}\right)$; $\tau_{2}\left(\lambda_{2}, \nu_{2}\right)=\inf \left\{t>0 ; y_{t}^{-}\left(\lambda_{2}\right) \geq \nu_{2}\right\}$.

That is, $\tau_{1}\left(\lambda_{1}, \nu_{1}\right)$ and $\tau_{2}\left(\lambda_{2}, \nu_{2}\right)$ are the first times that the processes $y_{t}^{+}\left(\lambda_{1}\right)$ and $y_{t}^{-}\left(\lambda_{2}\right)$ reach their corresponding thresholds $\nu_{1}$ and $\nu_{2}$ respectively. The modified drift 2-CUSUM stopping rules are then of the form $\tau\left(\lambda_{1}, \lambda_{2}, \nu_{1}, \nu_{2}\right)=\tau_{1}\left(\lambda_{1}, \nu_{1}\right) \wedge \tau_{2}\left(\lambda_{2}, \nu_{2}\right)$.

In what follows we will focus on modified drift 2-CUSUM harmonic mean stopping rules which belong to the class $\mathcal{G}_{M}=\left\{\tau\left(\lambda_{1}, \lambda_{2}, \nu_{1}, \nu_{2}\right) ; \nu_{1}=\nu_{2}\right\}$.

We also define the following quantities, the use of which will become apparent later.

Definition 2.3 For $a>0$ and $b>0$, we define

1. $U^{+}(a)=\inf \left\{t>0 ; u_{t}^{+} \geq a\right\}$,

2. $U^{-}(b)=\inf \left\{t>0 ;-u_{t}^{-} \leq-b\right\}$, and

3. $\Pi(a, b)=P\left(U^{+}(a)<U^{-}(b)\right)$.

In the sequel we will repeatedly use the indices $i, j \in\{1,2\}$ and the function

$$
f_{\nu}(y)=\frac{e^{y \nu}-y \nu-1}{y^{2}} .
$$

According to Hadjiliadis and Moustakides (2006), Siegmund (1985), and Taylor (1975) we have

$$
\begin{aligned}
E_{\infty}\left(\tau_{i}\left(\nu_{i}\right)\right) & =2 f_{\nu_{i}}\left(\mu_{i}\right), i=1,2, \\
E_{0}^{i}\left(\tau_{i}\left(\nu_{i}\right)\right) & =2 f_{\nu_{i}}\left(-\mu_{i}\right), i=1,2, \\
E_{0}^{i}\left(\tau_{j}\left(\nu_{j}\right)\right) & =2 f_{\nu_{j}}\left(\mu_{j}+2 \mu_{i}\right), \quad i \neq j, i, j \in\{1,2\} .
\end{aligned}
$$

Moreover, according to Hadjiliadis (2005) and Taylor (1975) we also have

$$
\begin{aligned}
E_{\infty}\left(\tau_{i}\left(\lambda_{i}, \nu\right)\right) & =2 f_{\nu}\left(\lambda_{i}\right), \\
E_{0}^{i}\left(\tau_{i}\left(\lambda_{i}, \nu\right)\right) & =2 f_{\nu}\left(\lambda_{i}-2 \mu_{i}\right), \\
E_{0}^{i}\left(\tau_{j}\left(\lambda_{j}, \nu\right)\right) & =2 f_{\nu}\left(\lambda_{j}+2 \mu_{i}\right) .
\end{aligned}
$$

For any 2-CUSUM stopping rule $\tau$ it is true that $J_{L}(\tau)=\max \left\{E_{0}^{1}[\tau], E_{0}^{2}[\tau]\right\}$ (see Hadjiliadis and Moustakides, 2006; Hadjiliadis and Poor, 2008). Thus, equation (2.4) takes the form

$$
E_{0}^{1}[\tau]=E_{0}^{2}[\tau]
$$




\section{THE FIRST MOMENT OF A GENERAL 2-CUSUM RULE}

We begin with our main expression for the first moment of a general classical 2-CUSUM stopping rule $\tau\left(\nu_{1}, \nu_{2}\right)$. To simplify the expressions that follow we introduce

$$
\begin{aligned}
\alpha_{j}(r, \xi) & =\exp \left\{-(r-1) \nu_{j}\left(r\left(\mu_{1}+\mu_{2}\right)+\mu_{j}-\xi\right)\right\} \\
\beta_{j}(r, \xi) & =\exp \left\{-r \nu_{j}\left(r\left(\mu_{1}+\mu_{2}\right)+\mu_{i}-\xi\right)\right\} \\
A_{i}(\xi) & =\sum_{r=1}^{\infty}\left[\left(r\left[r\left(\mu_{1}+\mu_{2}\right)+\mu_{i}-\xi\right]\right)\left(\alpha_{i}(r, \xi)-\alpha_{i}(r+1, \xi)\right)\right] \\
B_{i}(\xi) & =\sum_{r=1}^{\infty}\left[\left(r\left(\mu_{1}+\mu_{2}\right)+\frac{1}{2}\left(\mu_{i}-\xi\right)\right) \beta_{i}(r, \xi)\right]
\end{aligned}
$$

Theorem 3.1 Let $\tau\left(\nu_{1}, \nu_{2}\right)=\tau_{1}\left(\nu_{1}\right) \wedge \tau_{2}\left(\nu_{2}\right)$ be any 2-CUSUM stopping rule and denote $\tau\left(\nu_{1}, \nu_{2}\right)$ by $\tau$. Moreover, let $f$ be as in (2.5). Then, for $\nu_{i} \geq \nu_{j}, i \neq j$, with $\Delta=\nu_{i}-\nu_{j}$, we have

$$
\begin{aligned}
E_{0}^{i}[\tau] & =2 f_{\nu_{j}}\left(\mu_{j}+2 \mu_{i}\right)\left[1-\frac{f_{\nu_{j}}\left(\mu_{j}+2 \mu_{i}\right)}{f_{\nu_{j}}\left(-\mu_{i}\right)+f_{\nu_{j}}\left(\mu_{j}+2 \mu_{i}\right)} e^{-2 \Delta \cdot B_{i}(0)}\right], \\
E_{\infty}[\tau] & =2 f_{\nu_{j}}\left(\mu_{j}\right)\left[1-\frac{f_{\nu_{j}}\left(\mu_{j}\right)}{f_{\nu_{j}}\left(\mu_{j}\right)+f_{\nu_{j}}\left(\mu_{i}\right)} e^{-2 \Delta \cdot B_{i}\left(2 \mu_{i}\right)}\right], \text { for } i=2, \\
E_{0}^{j}[\tau] & =2 f_{\nu_{j}}\left(-\mu_{j}\right)\left[1-\frac{f_{\nu_{j}}\left(-\mu_{j}\right)}{f_{\nu_{j}}\left(-\mu_{j}\right)+f_{\nu_{j}}\left(\mu_{i}+2 \mu_{j}\right)} e^{-\Delta \cdot A_{j}(0)}\right], \\
E_{\infty}[\tau] & =2 f_{\nu_{j}}\left(\mu_{j}\right)\left[1-\frac{f_{\nu_{j}}\left(\mu_{j}\right)}{f_{\nu_{j}}\left(\mu_{j}\right)+f_{\nu_{j}}\left(\mu_{i}\right)} e^{-\Delta \cdot A_{j}\left(2 \mu_{j}\right)}\right], \text { for } j=2 .
\end{aligned}
$$

We notice that for any $\tau$ with $\nu_{1}=\nu_{2}$, all of the above expressions reduce to the well-known harmonic mean rule (see Siegmund, 1985). That is, for $\nu_{1}=\nu_{2}$, under any measure, we obtain

$$
E[\tau]=\frac{E\left[\tau_{1}\right] E\left[\tau_{2}\right]}{E\left[\tau_{1}\right]+E\left[\tau_{2}\right]}
$$

Moreover, it can easily be seen from the expressions of Theorem 3.1 that the harmonic mean rule holds as a lower bound to the first moment of a general classical 2-CUSUM stopping rule (see e.g. Dragalin, 1997).

In order to prove Theorem 3.1 we will need to make use of two preliminary results that are summarized in the following two lemmas.

Lemma 3.1 We have

$$
\sup _{s \leq t}\left(y_{s}^{+}+y_{s}^{-}\right)=\max \left\{\sup _{s \leq t} y_{s}^{+}, \sup _{s \leq t} y_{s}^{-}\right\}
$$

Proof. Observe that $y_{t}^{+}+y_{t}^{-}=-\frac{1}{2}\left(\mu_{1}+\mu_{2}\right) t-m_{t}^{+}-m_{t}^{-}$. We notice that the process $y_{t}^{+}+y_{t}^{-}$can only increase when either $u_{t}^{+}=m_{t}^{+}$or $u_{t}^{-}=m_{t}^{-}$, both of which cannot happen at the same time, since that would imply that $y_{t}^{+}+y_{t}^{-}$is 0 . Therefore, $y_{t}^{+}+y_{t}^{-}$ 
is a strictly decreasing function of time unless either $y_{t}^{+}=0$ or $y_{t}^{-}=0$, at which instant $\max \left\{y_{t}^{+}, y_{t}^{-}\right\}=\sup _{s \in[0, t]}\left\{\max \left\{y_{s}^{+}, y_{s}^{-}\right\}\right\}$.

As a consequence of Lemma 3.1, we have that

$$
\begin{aligned}
& \nu_{2} \geq \nu_{1} \Rightarrow\left\{\tau_{2}<\tau_{1}\right\} \quad \subseteq\left\{y_{\tau_{2}}^{+}=0\right\} \\
& \nu_{1} \geq \nu_{2} \Rightarrow\left\{\tau_{1}<\tau_{2}\right\} \quad \subseteq\left\{y_{\tau_{1}}^{-}=0\right\}
\end{aligned}
$$

Remark 3.1 We notice that in the case of Khan's (2007) stopping rule $T=T_{1}\left(h_{1}\right) \wedge T_{2}\left(h_{2}\right)$ (see Remark 2.1), equations (3.10) and (3.11) become respectively

$$
\begin{aligned}
& h_{2} \geq h_{1} \Rightarrow\left\{T_{2}<T_{1}\right\} \equiv\left\{Y_{T_{2}}^{+}=0\right\} \\
& h_{1} \geq h_{2} \Rightarrow\left\{T_{1}<T_{2}\right\} \equiv\left\{Y_{T_{1}}^{-}=0\right\} .
\end{aligned}
$$

Similarly to Khan's (1981) Lemma 2 and Khan's (1985) Lemma 1 in which (3.12) and (3.13) are used, we have the following lemma which uses (3.10) and (3.11) instead.

Lemma 3.2 Let $\tau_{1}$ and $\tau_{2}$ be the one-sided CUSUM stopping branches of $\tau_{\mathcal{G}} \in \mathcal{G}$ having the same threshold parameter $\nu$. We then have

$$
P\left(\tau_{2}<\tau_{1}\right)=\frac{E\left[\tau_{1}\right]}{E\left[\tau_{1}\right]+E\left[\tau_{2}\right]}, P\left(\tau_{1}<\tau_{2}\right)=\frac{E\left[\tau_{2}\right]}{E\left[\tau_{1}\right]+E\left[\tau_{2}\right]} .
$$

Proof. For simplicity in this proof we will use $\tau$ to denote $\tau_{\mathcal{G}}$. We have $\tau=\tau_{1} \wedge \tau_{2}$. Hence,

$$
\tau_{1}=\tau+\left(\tau_{1}-\tau\right)^{+}, \tau_{2}=\tau+\left(\tau_{2}-\tau\right)^{+} .
$$

Conditioning on $\left\{\tau=\tau_{2}\right\}$, and subsequently on its complement, and taking expectations we have

$$
\begin{aligned}
& E\left[\tau_{1}\right]=E[\tau]+E\left[\tau_{1}-\tau_{2} \mid \tau_{2}<\tau_{1}\right] \cdot P\left(\tau_{2}<\tau_{1}\right), \\
& E\left[\tau_{2}\right]=E[\tau]+E\left[\tau_{2}-\tau_{1} \mid \tau_{1}<\tau_{2}\right] \cdot P\left(\tau_{1}<\tau_{2}\right) .
\end{aligned}
$$

Since $\tau_{1}$ and $\tau_{2}$ have the same thresholds $\nu$, using (3.10) and (3.11), we obtain

$$
\left\{\tau_{2}<\tau_{1}\right\} \subseteq\left\{y_{\tau_{2}}^{+}=0\right\},\left\{\tau_{1}<\tau_{2}\right\} \subseteq\left\{y_{\tau_{1}}^{-}=0\right\}
$$

Therefore, (3.14) and (3.15) become

$$
E\left[\tau_{1}\right]=E[\tau]+E\left[\tau_{1}\right] \cdot P\left(\tau_{2}<\tau_{1}\right), E\left[\tau_{2}\right]=E[\tau]+E\left[\tau_{2}\right] \cdot P\left(\tau_{1}<\tau_{2}\right),
$$

from which, by eliminating $E[\tau]$, the result follows. 
Proof of Theorem 3.1. Let us suppose that $\nu_{1}>\nu_{2}$. Then, using (3.11) and (3.15), we have that

$$
E\left[\tau_{2}\left(\nu_{2}\right)\right]=E[\tau]+E\left[\tau_{2}\left(\nu_{2}\right)\right] \cdot P\left(\tau_{1}\left(\nu_{1}\right)<\tau_{2}\left(\nu_{2}\right)\right)
$$

Hence,

$$
E[\tau]=E\left[\tau_{2}\left(\nu_{2}\right)\right] \cdot P\left(\tau_{2}\left(\nu_{2}\right)<\tau_{1}\left(\nu_{1}\right)\right)
$$

We now proceed to express $P\left(\tau_{2}\left(\nu_{2}\right)<\tau_{1}\left(\nu_{1}\right)\right)$ in terms of $\Pi(a, b)$ as it appears in Definition 2.3. Notice that we can rewrite the probability of its complement, namely $P\left(\tau_{1}\left(\nu_{1}\right)<\right.$ $\left.\tau_{2}\left(\nu_{2}\right)\right)^{2}$, as

$$
P\left(\tau_{1}\left(\nu_{1}\right)<\tau_{2}\left(\nu_{2}\right)\right)=P\left(\tau_{1}\left(\nu_{1}\right)<\tau_{2}\left(\nu_{2}\right) \mid \tau_{1}\left(\nu_{2}\right)<\tau_{2}\left(\nu_{2}\right)\right) P\left(\tau_{1}\left(\nu_{2}\right)<\tau_{2}\left(\nu_{2}\right)\right) .
$$

Using Lemma 3.2, however, with $\nu=\nu_{2}$, we obtain

$$
P\left(\tau_{1}\left(\nu_{2}\right)<\tau_{2}\left(\nu_{2}\right)\right)=\frac{E\left[\tau_{2}\left(\nu_{2}\right)\right]}{E\left[\tau_{1}\left(\nu_{2}\right)\right]+E\left[\tau_{2}\left(\nu_{2}\right)\right]}
$$

To get an expression for

$$
P\left(\tau_{1}\left(\nu_{1}\right)<\tau_{2}\left(\nu_{2}\right) \mid \tau_{1}\left(\nu_{2}\right)<\tau_{2}\left(\nu_{2}\right)\right)
$$

we first consider the dynamics of $y_{t}^{+}$and $y_{t}^{-}$under all relevant measures. Using Definition 2.1 , we can write $y_{t}^{+}=u_{t}^{+}-m_{t}^{+}$and $y_{t}^{-}=u_{t}^{-}-m_{t}^{-}$where the dynamics of $u_{t}^{+}$and $-u_{t}^{-}$ are summarized in the following table:

Table 2. The dynamics of $u_{t}^{+}$and $-u_{t}^{-}$under the different regimes

\begin{tabular}{|l|l|l|l|}
\hline & no change $\left(P_{\infty}\right)$ & change is $\mu_{1}\left(P_{0}^{1}\right)$ & change is $-\mu_{2}\left(P_{0}^{2}\right)$ \\
\hline$u_{t}^{+}$ & $w_{t}-\frac{1}{2} \mu_{1} t$ & $w_{t}+\frac{1}{2} \mu_{1} t$ & $w_{t}-\frac{1}{2}\left(\mu_{1}+2 \mu_{2}\right) t$ \\
\hline$-u_{t}^{-}$ & $w_{t}+\frac{1}{2} \mu_{2} t$ & $w_{t}+\frac{1}{2}\left(\mu_{2}+2 \mu_{1}\right) t$ & $w_{t}-\frac{1}{2} \mu_{2} t$ \\
\hline
\end{tabular}

\footnotetext{
${ }^{2}$ Notice that $P\left(\tau_{1}\left(\nu_{1}\right)=\tau_{2}\left(\nu_{2}\right)\right)=0$ under any measure and any $\nu_{1}>0$ and $\nu_{2}>0$.
} 
Therefore, if we were to divide the interval $\left[0, \nu_{1}-\nu_{2}\right]$ into $n$ equal length intervals, then

$$
P\left(\tau_{1}\left(\nu_{1}\right)<\tau_{2}\left(\nu_{2}\right) \mid \tau_{1}\left(\nu_{2}\right)<\tau_{2}\left(\nu_{2}\right)\right)=\lim _{n \rightarrow \infty} P\left(\cap_{i=1}^{n} E_{i}\right),
$$

where $E_{i}$ is the event that $\left\{\sup _{\left\{t \in I_{i}\right\}} y_{t}^{-}<\nu_{2}\right\}$ conditioned upon the initial value of $y_{t}^{-}$at the left endpoint of each interval $I_{i}$ being equal to 0 and $\left\{I_{i}\right\}$ are the random intervals

$$
I_{i}=\left[\tau_{1}\left(\nu_{2}+(i-1) \frac{\left(\nu_{1}-\nu_{2}\right)}{n}\right), \tau_{1}\left(\nu_{2}+i \frac{\left(\nu_{1}-\nu_{2}\right)}{n}\right)\right] .
$$

Because of the strong Markov property of Brownian motion the events $\left\{E_{i}\right\}$ are independent, equiprobable and they have the same probability as the event

$$
\left\{\begin{array}{c}
\text { The process } u_{t}^{+} \text {increases by at least } \frac{\nu_{1}-\nu_{2}}{n} \text { units } \\
\text { before the process }-u_{t}^{-} \text {falls by } \nu_{2} \text { (or more) units }
\end{array}\right\} \text {. }
$$

A depiction of the strong Markov property is given in Fig. 1, where the upper solid black line represents the process $-u_{t}^{-}=\xi_{t}+\frac{1}{2} \mu_{2} t$, the upper dashed line represents its running supremum, (that is, the process $\sup _{s \leq t}\left(\xi_{s}+\frac{1}{2} \mu_{2} s\right)=-m_{t}^{-}$), the lower solid black line represents the process $u_{t}^{+}=\xi_{t}-\frac{1}{2} \mu_{1} t$ and the lower dashed line its running infimum (that is, the process $m_{t}^{+}=\inf _{s \leq t}\left(\xi_{s}-\frac{1}{2} \mu_{1} s\right)$ ). We remark that the normalized CUSUM process $y_{t}^{-}$ is the difference between the upper dashed and the black lines and the normalized CUSUM process $y_{t}^{+}$is the difference between the lower solid black and the lower dashed lines (see Definition 2.1). The strong Markov property is depicted by a shift of the axis to the time point $\tau_{1}\left(\nu_{2}\right)$, that is the first time point at which the process $y_{t}^{+}$reaches $\nu_{2}$ (the difference between the lower solid black and the lower dashed lines is $\nu_{2}$ ). Representation (3.19) comes as a result of repeated such "shifts" of the axis (which are valid by the strong Markov property of Brownian motion) at each of the points $\tau_{1}\left(\nu_{2}+\frac{\nu_{1}-\nu_{2}}{n}\right), \tau_{1}\left(\nu_{2}+2 \frac{\nu_{1}-\nu_{2}}{n}\right), \ldots$, $\tau_{1}\left(\nu_{2}+(n-1) \frac{\nu_{1}-\nu_{2}}{n}\right)$.

Therefore, in view of Definition 2.3 and (3.20), (3.19) becomes

$$
P\left(\tau_{1}\left(\nu_{1}\right)<\tau_{2}\left(\nu_{2}\right) \mid \tau_{1}\left(\nu_{2}\right)<\tau_{2}\left(\nu_{2}\right)\right)=\lim _{n \rightarrow \infty} P\left(E_{1}\right)^{n}=\lim _{n \rightarrow \infty} \Pi\left(\frac{\nu_{1}-\nu_{2}}{n}, \nu_{2}\right)^{n} .
$$

Using Theorem 4.1 of Anderson (1960), we obtain the following representations for

$$
\lim _{n \rightarrow \infty} P\left(E_{1}\right)^{n}
$$

under the following different measures:

1. under $P_{0}^{1}$

$$
\begin{aligned}
\Pi\left(\frac{\nu_{1}-\nu_{2}}{n}, \nu_{2}\right)^{n}= & \left(1-\sum_{r=1}^{\infty}\left[e^{-2\left[(r-1)^{2} \gamma_{1} \delta_{1}+r^{2} \gamma_{2} \delta_{2}-r(r-1)\left(\gamma_{1} \delta_{2}+\gamma_{2} \delta_{1}\right)\right]}\right.\right. \\
& \left.\left.-e^{-2\left[r^{2}\left(\gamma_{1} \delta_{1}+\gamma_{2} \delta_{2}\right)-r(r+1) \gamma_{1} \delta_{2}-r(r-1) \gamma_{2} \delta_{1}\right]}\right]\right)^{n},
\end{aligned}
$$

where $\gamma_{1}=\frac{\nu_{1}-\nu_{2}}{n}, \gamma_{2}=-\nu_{2}, \delta_{1}=-\frac{1}{2} \mu_{1}$ and $\delta_{2}=-\frac{1}{2}\left(\mu_{2}+2 \mu_{1}\right)$. 


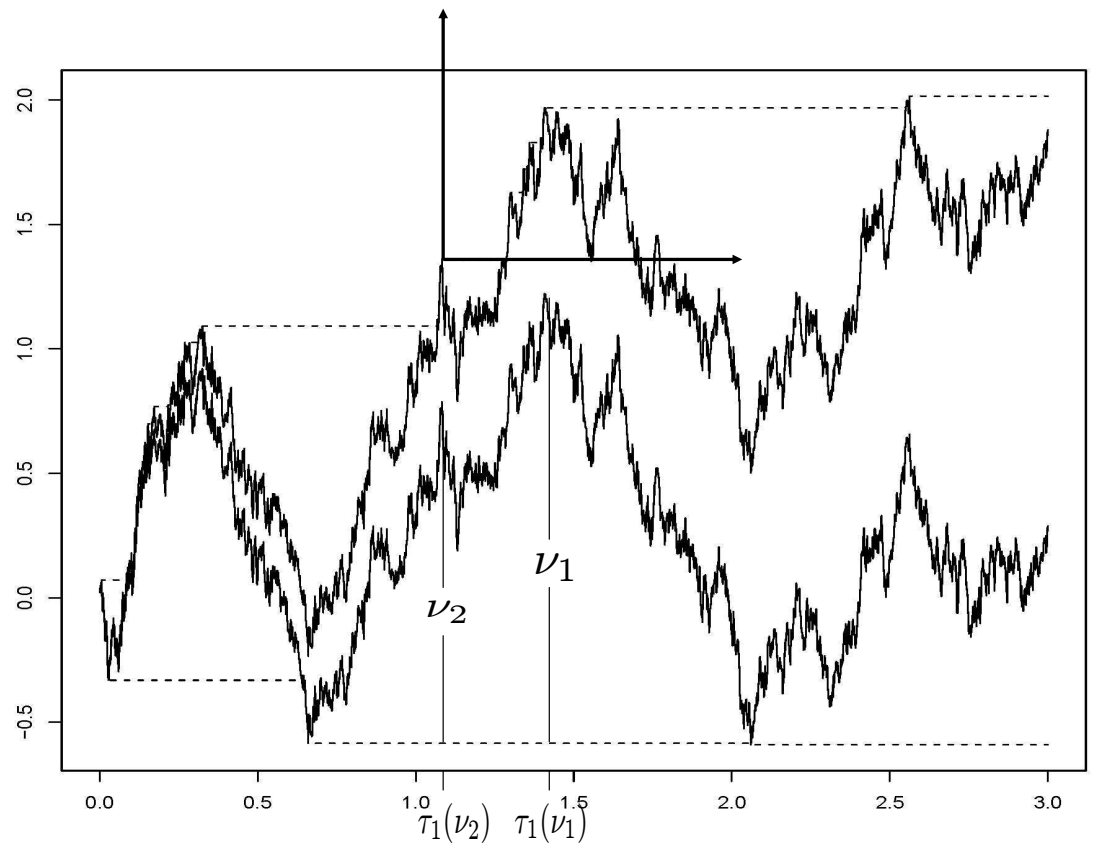

Figure 1. A demonstration of the strong Markov property of Brownian motion.

2. under $P_{0}^{2}$

$$
\begin{aligned}
\Pi\left(\frac{\nu_{1}-\nu_{2}}{n}, \nu_{2}\right)^{n}= & \left(\sum _ { r = 1 } ^ { \infty } \left[e^{-2\left[r^{2} \gamma_{1} \delta_{1}+(r-1)^{2} \gamma_{2} \delta_{2}-r(r-1)\left(\gamma_{1} \delta_{2}+\gamma_{2} \delta_{1}\right)\right]}\right.\right. \\
& \left.\left.-e^{-2\left[r^{2}\left(\gamma_{1} \delta_{1}+\gamma_{2} \delta_{2}\right)-r(r-1) \gamma_{1} \delta_{2}-r(r+1) \gamma_{2} \delta_{1}\right]}\right]\right)^{n}
\end{aligned}
$$

where $\gamma_{1}=\frac{\nu_{1}-\nu_{2}}{n}, \gamma_{2}=-\nu_{2}, \delta_{1}=\frac{1}{2}\left(\mu_{1}+2 \mu_{2}\right)$ and $\delta_{2}=\frac{1}{2} \mu_{2}$.

3. under $P_{\infty}$

$\Pi\left(\frac{\nu_{1}-\nu_{2}}{n}, \nu_{2}\right)^{n}$ has the same representation as in (3.23), for $\delta_{1}=\frac{1}{2} \mu_{1}$ and $\delta_{2}=-\frac{1}{2} \mu_{2}$.

Let

$$
\begin{aligned}
& C(r)=\exp \left(-2\left(r^{2} \gamma_{2} \delta_{2}-r(r-1) \gamma_{2} \delta_{1}\right)\right) \\
& D(r)=\exp \left(-2 \gamma_{1}\left[r^{2} \delta_{1}-r(r-1) \delta_{2}\right]\right) .
\end{aligned}
$$

Factoring out the term $C(r)$, the right-hand side of (3.22) becomes

$$
\left(1-\sum_{r=1}^{\infty} C(r)\left[e^{-2 \gamma_{1}\left[(r-1)^{2} \delta_{1}-r(r-1) \delta_{2}\right]}-e^{-2 \gamma_{1}\left[r^{2} \delta_{1}-r(r+1) \delta_{2}\right]}\right]\right)^{n} .
$$


Since $\gamma_{1}=\frac{\nu_{1}-\nu_{2}}{n}$ and $\gamma_{2}=-\nu_{2}$, we have that

$$
\begin{aligned}
e^{-2 \gamma_{1}\left[(r-1)^{2} \delta_{1}-r(r-1) \delta_{2}\right]}-e^{-2 \gamma_{1}\left[r^{2} \delta_{1}-r(r+1) \delta_{2}\right]} & \\
= & \left(1-\frac{2\left(\nu_{1}-\nu_{2}\right)}{n}\left[(r-1)^{2} \delta_{1}-r(r-1) \delta_{2}\right]+o\left(\frac{1}{n}\right)\right) \\
& -\left(1-\frac{2\left(\nu_{1}-\nu_{2}\right)}{n}\left[r^{2} \delta_{1}-r(r+1) \delta_{2}\right]+o\left(\frac{1}{n}\right)\right) .
\end{aligned}
$$

Substituting (3.25) into (3.24), we obtain

$$
\begin{aligned}
& \lim _{n \rightarrow \infty}\left(1+\frac{2\left(\nu_{1}-\nu_{2}\right)}{n}\left[\sum_{r=1}^{\infty}\left(\delta_{1}+2 r\left(\delta_{2}-\delta_{1}\right) C(r)\right]\right)^{n}\right. \\
& \left.\quad=\exp \left\{-2\left(\nu_{1}-\nu_{2}\right)\left[\sum_{r=1}^{\infty}\left(\delta_{1}+2 r\left(\delta_{2}-\delta_{1}\right)\right) C(r)\right]\right\}\right],
\end{aligned}
$$

as claimed.

Similarly the right-hand side of (3.23) can be written as

$$
\left(\sum_{r=1}^{\infty} D(r)\left[e^{-2\left[(r-1)^{2} \gamma_{2} \delta_{2}-r(r-1) \gamma_{2} \delta_{1}\right]}-e^{-2\left[r^{2} \gamma_{2} \delta_{2}-r(r+1) \gamma_{2} \delta_{1}\right]}\right]\right)^{n}
$$

Observe that since $\gamma_{1}=\frac{\nu_{1}-\nu_{2}}{n}$,

$$
\begin{aligned}
D(r) & =e^{-2 \gamma_{1}\left[r^{2} \delta_{1}-r(r-1) \delta_{2}\right]}=\exp \left\{-\frac{2\left(\nu_{1}-\nu_{2}\right)}{n}\left[r^{2} \delta_{1}-r(r-1) \delta_{2}\right]\right\} \\
& =1-\frac{2\left(\nu_{1}-\nu_{2}\right)}{n}\left[r^{2} \delta_{1}-r(r-1) \delta_{2}\right]+o\left(\frac{1}{n}\right) .
\end{aligned}
$$

Moreover,

$$
\sum_{r=1}^{\infty}\left[e^{-2\left[(r-1)^{2} \gamma_{2} \delta_{2}-r(r-1) \gamma_{2} \delta_{1}\right]}-e^{-2\left[r^{2} \gamma_{2} \delta_{2}-r(r+1) \gamma_{2} \delta_{1}\right]}\right]=1
$$

which is recognizable as quantity $P_{1}$ in Theorem 4.1 of Anderson (1960) for $\gamma_{1}=0$. Indeed it is with probability 1 that a Brownian process, which starts at zero, will first hit the boundary $\delta_{1} t$, before hitting the linear boundary $\gamma_{2}+\delta_{2} t$, since this occurs at time $t=0$.

Thus,

$\lim _{n \rightarrow \infty} \Pi\left(\frac{\nu_{1}-\nu_{2}}{n}, \nu_{2}\right)^{n}=\lim _{n \rightarrow \infty}\left(1-\frac{2 \Delta}{n}\left[\sum_{r=1}^{\infty}\left[r^{2} \delta_{1}-r(r-1) \delta_{2}\right]\left[\alpha_{2}(r, 0)-\alpha_{2}(r+1,0)\right]\right]\right)^{n}$

which reduces to

$$
\exp \left\{-\Delta A_{2}(0)\right\}
$$

as claimed.

All other cases follow similarly. 


\section{THE SYMMETRIC CASE}

In this section we treat the case that $\mu_{1}=\mu_{2}=\mu$. The best 2-CUSUM stopping rule has to satisfy (2.12). Moreover, the best amongst the classical 2-CUSUM stopping rules, in the symmetric case, belongs to the class $\mathcal{G}$ of harmonic mean 2-CUSUM rules and is also unique within its class (see Hadjiliadis and Poor, 2008). Furthermore, in Hadjiliadis (2005) it is seen that (2.12) is satisfied for all modified drift 2-CUSUM stopping rules of class $\mathcal{G}_{M}$ for which

$$
\lambda_{1}-\lambda_{2}=2\left(\mu_{1}-\mu_{2}\right) .
$$

Notice that equation (4.1) implies that $\lambda_{1}$ should be equal to $\lambda_{2}$ whenever there is a symmetric change, namely $\mu_{1}=\mu_{2}$. The optimality of the modified drift 2-CUSUM harmonic mean rules within the class of all modified drift 2-CUSUM harmonic mean rules $\mathcal{G}_{M}$ (see Hadjiliadis, 2005) for any value of $\gamma$, suggests that one should compare the performance of the classical 2-CUSUM harmonic mean rule with the performance of the modified drift 2-CUSUM harmonic mean rule for the same mean time to false alarms $\gamma$. Since the latter involves one more free parameter, namely $\lambda=\lambda_{1}=\lambda_{2}$, over which minimization of $J_{L}(\tau)$ can take place, it is expected that it will have a strictly better performance than its classical 2-CUSUM counterpart. It is also expected, due to the asymptotic optimality of the classical 2-CUSUM harmonic mean rule as $\gamma \rightarrow \infty$ (see Hadjiliadis and Moustakides, 2006), that the free parameter $\lambda$ which minimizes $J_{L}(\tau)$ converges to $\mu=\mu_{1}=\mu_{2}$ as $\gamma \rightarrow \infty$.

In particular, for the modified parameter 2-CUSUM $\tau(\lambda, \nu)$, using (2.9), (2.10), (2.11) and the harmonic mean rule (3.9), (2.2) becomes

$$
\begin{gathered}
\min _{\lambda}\left\{2 \frac{f_{\nu}(\lambda+2 \mu) f_{\nu}(\lambda-2 \mu)}{f_{\nu}(\lambda+2 \mu)+f_{\nu}(\lambda-2 \mu)}\right\} \\
\text { subject to } f_{\nu}(\lambda)=\gamma .
\end{gathered}
$$

From the above constraint it follows that as $\gamma \rightarrow \infty$ we obtain

$$
\lambda \nu=\log (\gamma)(1+o(1))
$$

By inspection of the delay function

$$
E_{0}^{1}[\tau(\lambda, \nu)]=E_{0}^{2}[\tau(\lambda, \nu)]=2 \frac{f_{\nu}(\lambda+2 \mu) f_{\nu}(\lambda-2 \mu)}{f_{\nu}(\lambda+2 \mu)+f_{\nu}(\lambda-2 \mu)},
$$

it is easily seen that the minimal detection delay as $\nu$ increases, occurs for $\lambda<2 \mu$ (see Theorem 2 of Hadjiliadis and Moustakides, 2006) and becomes

$$
2 \frac{f_{\nu}(\lambda+2 \mu) f_{\nu}(\lambda-2 \mu)}{f_{\nu}(\lambda+2 \mu)+f_{\nu}(\lambda-2 \mu)}=\frac{2 \nu}{2 \mu-\lambda}(1+o(1)) .
$$

Substituting (4.3) into (4.5), we obtain

$$
\frac{f_{\nu}(\lambda+2 \mu) f_{\nu}(\lambda-2 \mu)}{f_{\nu}(\lambda+2 \mu)+f_{\nu}(\lambda-2 \mu)}=\frac{2 \log \gamma}{\lambda(2 \mu-\lambda)}(1+o(1))
$$



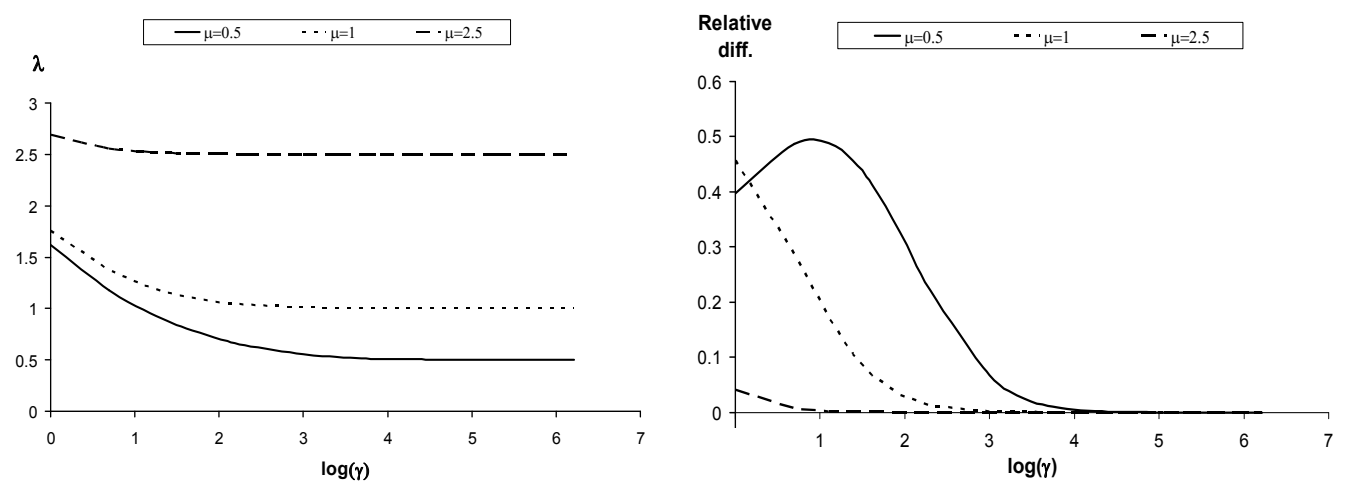

Figure 2. (Left) Convergence of $\lambda$ as a function of $\log (\gamma)$. The solid curve corresponds to $\mu=0.5$, the dotted curve to $\mu=1$, and the dashed curve to $\mu=2.5$. (Right) Relative diff., defined in (4.7), as a function of $\log (\gamma)$. Solid, dotted, and dashed curves same as in (Left).

which is minimized for the choice $\lambda=\mu$.

This asserts that the best asymptotically in the class $\mathcal{G}_{M}$ is the same as the best in the class $\mathcal{G}$.

For the purpose of clarity let us denote by $\mathrm{DD}_{\text {modified }}$ the quantity of (4.4) and by $\mathrm{DD}_{\text {classical }}$ the same quantity for $\lambda=\mu$. In Fig. 2 (Right side), the relative difference of these two quantities, namely

$$
\text { Relative diff. }=100 \times \frac{\mathrm{DD}_{\text {classical }}-\mathrm{DD}_{\text {modified }}}{\mathrm{DD}_{\text {classical }}},
$$

is plotted against $\log \gamma$.

Remark 4.1 It is possible to solve for $\nu$ in the equation $f_{\nu}(\lambda)=\gamma$ in terms of the Lambert $W$ function. In particular, let $W$ be the second real branch of the Lambert $\mathrm{W}$ function (see Corless et al., 1996), that is represented by the function ProductLog[-1, · ] in Mathematica (see Appendix A). Then,

$$
\nu=\frac{\log \left[-W\left(-e^{-\left(\lambda^{2} \gamma+1\right)}\right)\right]}{\lambda} .
$$

Optimization for each value of $\gamma$ can then be carried out by substituting for $\nu$ from (4.8) into (4.4).

The results found above are represented in Fig. 2. The percentage decrease (Relative diff. (4.7) ) in detection delay of the modified 2-CUSUM harmonic mean equalizer rule is barely noticeable and occurs only for small values of changes in the drift parameter $\mu$ and small values of $\gamma$. We also observe that this percentage decrease in the relative difference (4.7) is achieved for even smaller values of $\gamma$ as $\mu$ increases.

The results for the non-symmetric case are far more interesting and are summarized in the following section. 


\section{THE NON-SYMMETRIC CASE}

In this section we consider the case of a non-symmetric change $\mu_{1} \neq \mu_{2}$. Without loss of generality we assume that $\mu_{1}>\mu_{2}$. In Hadjiliadis and Poor (2008) it is seen that the best classical 2-CUSUM stopping rule is unique and satisfies $\nu_{1}>\nu_{2}$. We thus compare the detection delay $J_{L}(\cdot)$ of the unique classical 2-CUSUM stopping rule $\tau\left(\nu_{1}, \nu_{2}\right)$ with $\nu_{1}>\nu_{2}$ that satisfies (2.12) to that of the modified drift 2-CUSUM harmonic mean rule with $\lambda_{2}$ a free parameter, over which the detection delay $J_{L}(\cdot)$ is minimized. For the purpose of clarity let us denote the detection delay of the former 2-CUSUM by DD classical eq. and the detection delay of the latter 2-CUSUM by $\mathrm{DD}_{\text {modified optimized }}$. For the modified drift 2-CUSUM harmonic mean rule (4.1) implies that $\lambda_{1}=2\left(\mu_{1}-\mu_{2}\right)+\lambda_{2}$. Both rules are chosen so as to satisfy the false alarm constraint with equality (2.3).

Fig. 3 demonstrates the relationship of the ratio of thresholds $\frac{\nu_{1}}{\nu_{2}}$ as a function of $\log \gamma$ for the best classical 2-CUSUM stopping rule. The exact relationship can be extracted from
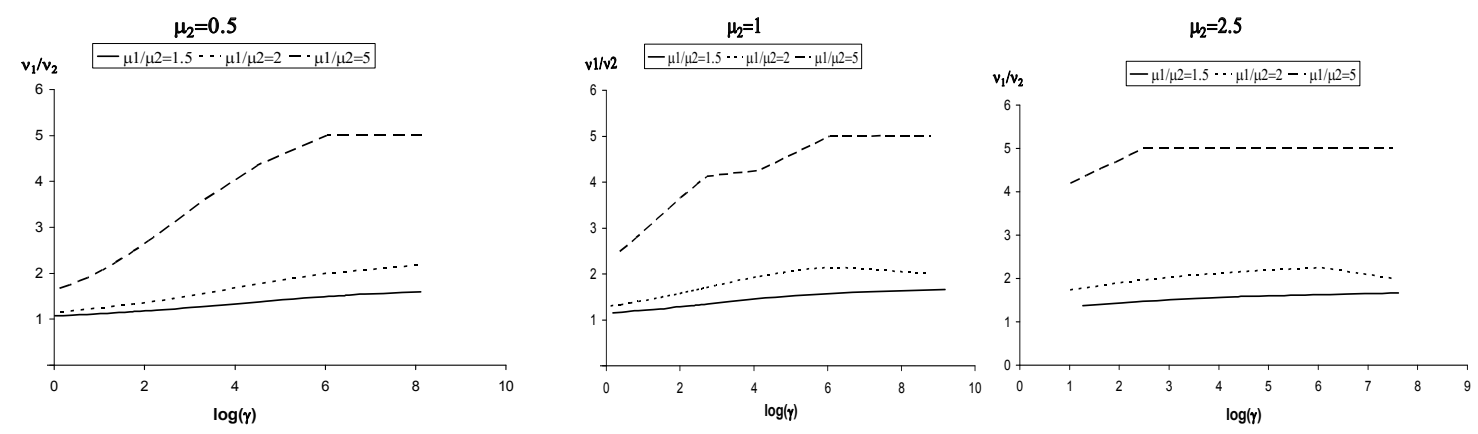

Figure 3. (Left) Case of $\mu_{2}=0.5$ : The ratio of thresholds $\frac{\nu_{1}}{\nu_{2}}$ of the best classical 2-CUSUM rule as a function of $\log (\gamma)$. The solid curve corresponds to $\frac{\mu_{1}}{\mu_{2}}=1.5$, the dotted curve to $\frac{\mu_{1}}{\mu_{2}}=2$, and the dashed curve to $\frac{\mu_{1}}{\mu_{2}}=5$. (Middle) Case of $\mu_{2}=1$. (Right) Case of $\mu_{2}=2.5$.

Theorem 3.1. In particular, $\nu_{1}$ and $\nu_{2}$ have to be chosen so that (2.12) holds. The first interesting feature is that the ratio $\frac{\nu_{1}}{\nu_{2}}$ increases as $\gamma \rightarrow \infty$. Another interesting characteristic that can be seen in Fig. 3, is that the ratio $\frac{\nu_{1}}{\nu_{2}}$ for which equation (2.12) holds is always less than the ratio $\frac{\mu_{1}}{\mu_{2}}$.

For the modified drift 2-CUSUM harmonic mean equalizer rule, the optimal choice of the free parameter $\lambda_{2}$ converges to $\mu_{2}$ (see Hadjiliadis and Moustakides, 2006). The modified drift 2-CUSUM harmonic mean equalizer rule is also asymptotically optimal as $\gamma \rightarrow \infty$ (see Hadjiliadis and Moustakides, 2006). Yet, in Fig. 4, it is seen that the modified drift 2-CUSUM harmonic mean equalizer rule has inferior performance to the classical 2-CUSUM equalizer rule. In particular, Fig. 4, displays the relative difference between the classical 2-CUSUM stopping rule that satisfies (2.12) to the modified drift 2-CUSUM harmonic mean rule with drift parameters $\lambda_{1}$ and $\lambda_{2}$ as suggested in the first paragraph; that is

$$
\text { Relative diff. }=100 \times \frac{\mathrm{DD}_{\text {modified optimized }}-\mathrm{DD}_{\text {classical eq. }}}{\mathrm{DD}_{\text {modified optimized }}} .
$$


It is seen that even for moderate values of $\gamma$ and any value of $\mu_{2}$ there is a clear percentage decrease in detection delay, as defined in (5.1), of the classical 2-CUSUM equalizer rule versus the modified drift 2-CUSUM harmonic mean equalizer rule. This relative difference reaches the level of $5 \%$ for $\log \gamma$ as high as $4, \mu_{2}=0.5$, and $\frac{\mu_{1}}{\mu_{2}}=1.5$ as seen in Fig. 4. This relative difference, however, decreases to 0 faster as $\frac{\mu_{1}}{\mu_{2}}$ increases.

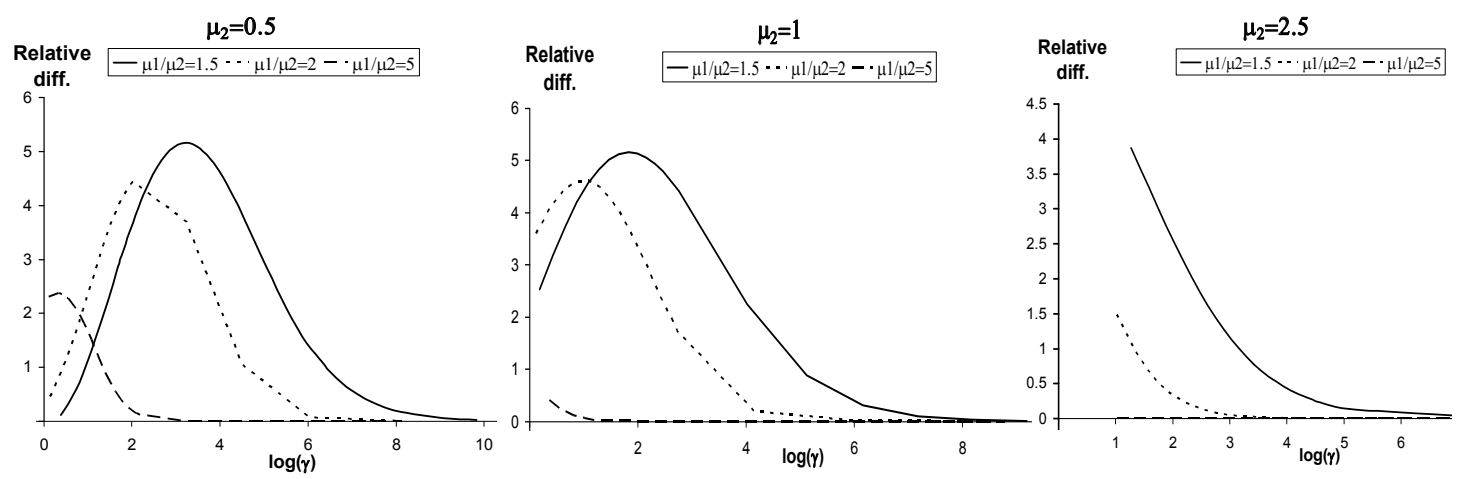

Figure 4. (Left) Case of $\mu_{2}=0.5$ : The relative difference between the modified 2-CUSUM and the classical 2-CUSUM equalizer rules is displayed as a function of $\log (\gamma)$. The solid curve corresponds to $\frac{\mu_{1}}{\mu_{2}}=1.5$, the dotted curve to $\frac{\mu_{1}}{\mu_{2}}=2$, and the dashed curve to $\frac{\mu_{1}}{\mu_{2}}=5$. (Middle) Case of $\mu_{2}=1$. (Right) Case of $\mu_{2}=2.5$. (Note that the dashed curve is very close zero, and thus hard to distinguish in the three graphs). Relative diff. is defined in (5.1).

In Fig. 4 it is also seen that the relative difference, defined in (5.1), tends to 0 as $\gamma \rightarrow \infty$. This is to be expected as both stopping rules display the same detection delay $J_{L}(\cdot)$, namely $\frac{2 \log (\gamma)}{\mu_{2}^{2}}(1+o(1))$, as $\gamma \rightarrow \infty$.

\section{DISCUSSION \& CONCLUDING REMARKS}

In this paper, we derive an exact closed form formula of the expected value of a general 2-CUSUM stopping rule in the Brownian motion model. This enables us to compare the performance of the modified drift 2-CUSUM harmonic mean equalizer rules, introduced for the first time in Hadjiliadis and Moustakides (2006), to the classical 2-CUSUM equalizer rules. As expected, in the symmetric case, the modified drift 2-CUSUM harmonic mean equalizer rules display a better performance than their classical 2-CUSUM counterparts (that is, a lesser detection delay for the same mean time to false alarms), since they introduce one more parameter (i.e. $\lambda$ ) over which minimization of the detection delay takes place. This gain however is not significant and is only seen in the case that $\mu$ is small and for small values of $\gamma$. The difference in their performance tends to 0 as $\gamma \rightarrow \infty$. On the other hand, in the case of a non-symmetric change, it is seen that even for moderate values of $\gamma$ the classical 2-CUSUM equalizer rule displays a better performance than its modified drift 2-CUSUM harmonic mean equalizer rule counterpart. This suggests that in the non-symmetric case it is more desirable to select a pair of thresholds for which (2.12) holds than to modify the drift parameters. Of course, the difference in the two detection 
delays tends to 0 as $\gamma \rightarrow \infty$ signifying that for large values of $\gamma$ it makes no difference in detection delay, which one of the two is selected.

The contribution of this paper, apart from the derivation of an exact closed form formula for the mean of a general 2-CUSUM stopping rules, is roughly summarized in the following table:

Table 3. Comparison of 2-CUSUM equalizer stopping rules

\begin{tabular}{|l|l|}
\hline Symmetric case & Non-symmetric case \\
\hline $\begin{array}{l}\text { Modified drift better than classical } \\
\text { harmonic mean rules for small values } \\
\text { of } \gamma \text { and } \mu\end{array}$ & $\begin{array}{l}\text { Classical non-harmonic rules better than } \\
\text { modified drift harmonic mean rules for almost } \\
\text { all values of } \gamma \text { and other parameters }\end{array}$ \\
\hline \multicolumn{2}{|c|}{ Difference tends to 0 as $\gamma \rightarrow \infty$} \\
\hline
\end{tabular}

Although, this paper concerns the case of a known two-sided post-change drift, we wish to add a comment on the unknown drift parameter case. Consider the case in which the drift assumed after the change is known to be two-sided and symmetric, namely + or $-\mu$ for some $\mu>0$ known to lie in a two-sided symmetric interval $I=[-M,-m] \cup[m, M]$, for some $M>m>0$. In Fig. 2, it is seen that there is a slight decrease in detection delay resulting from using a $\lambda>\mu$ for small values of $\gamma$, while as $\gamma \rightarrow \infty$ equation (4.6) implies that the detection delay is minimized for $\lambda=\mu$. But since we only have an interval of equally possible values for $\mu$ after the change, we can set $\mu=\frac{M+m}{2}$ and follow the minimization procedure of (4.2) in conjunction with Remark (4.1) to identify the optimal choice of $\lambda$. Similarly, consider the case in which the drift assumed after the changes is unknown but known to be non-symmetric and to thus lie in a non-symmetric interval $I^{\prime}=\left[-M_{2},-m_{2}\right] \cup\left[m_{1}, M_{1}\right]$ such that $\frac{m_{2}+M_{2}}{2}>\frac{m_{1}+M_{1}}{2}\left(\frac{m_{2}+M_{2}}{2}<\frac{m_{1}+M_{1}}{2}\right)$. It is then reasonable to set $\mu_{i}=\frac{m_{i}+M_{i}}{2}$ for $i=1,2$ and using the results in Section 5, choose $\nu_{2}>\nu_{1}$ $\left(\nu_{1}>\nu_{2}\right)$ so that (2.12) is satisfied. Finally, in the case that the interval of possible values is non-symmetric but with $\frac{m_{2}+M_{2}}{2}=\frac{m_{1}+M_{1}}{2}$, the same treatment as the one suggested in the symmetric case could be followed.

\section{APPENDIX A: SYMMETRIC CASE PROGRAM}

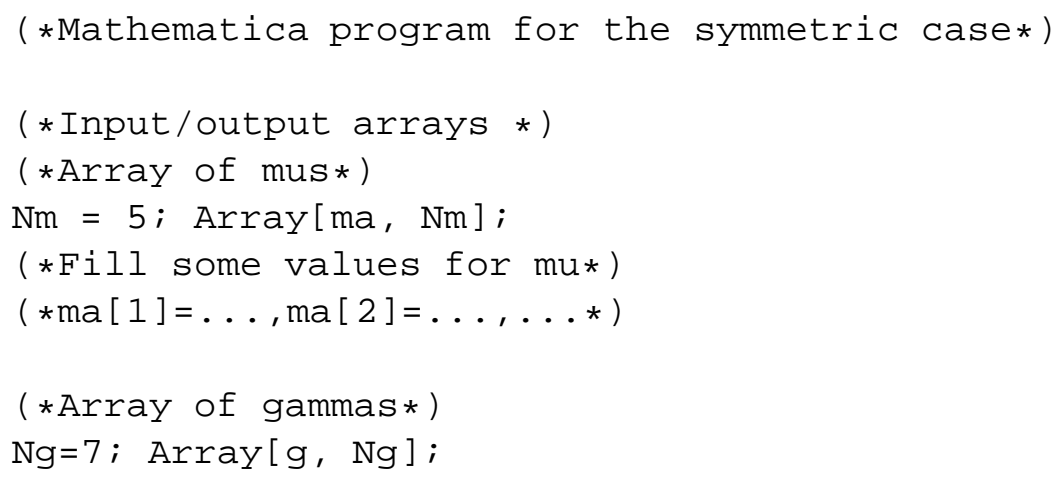




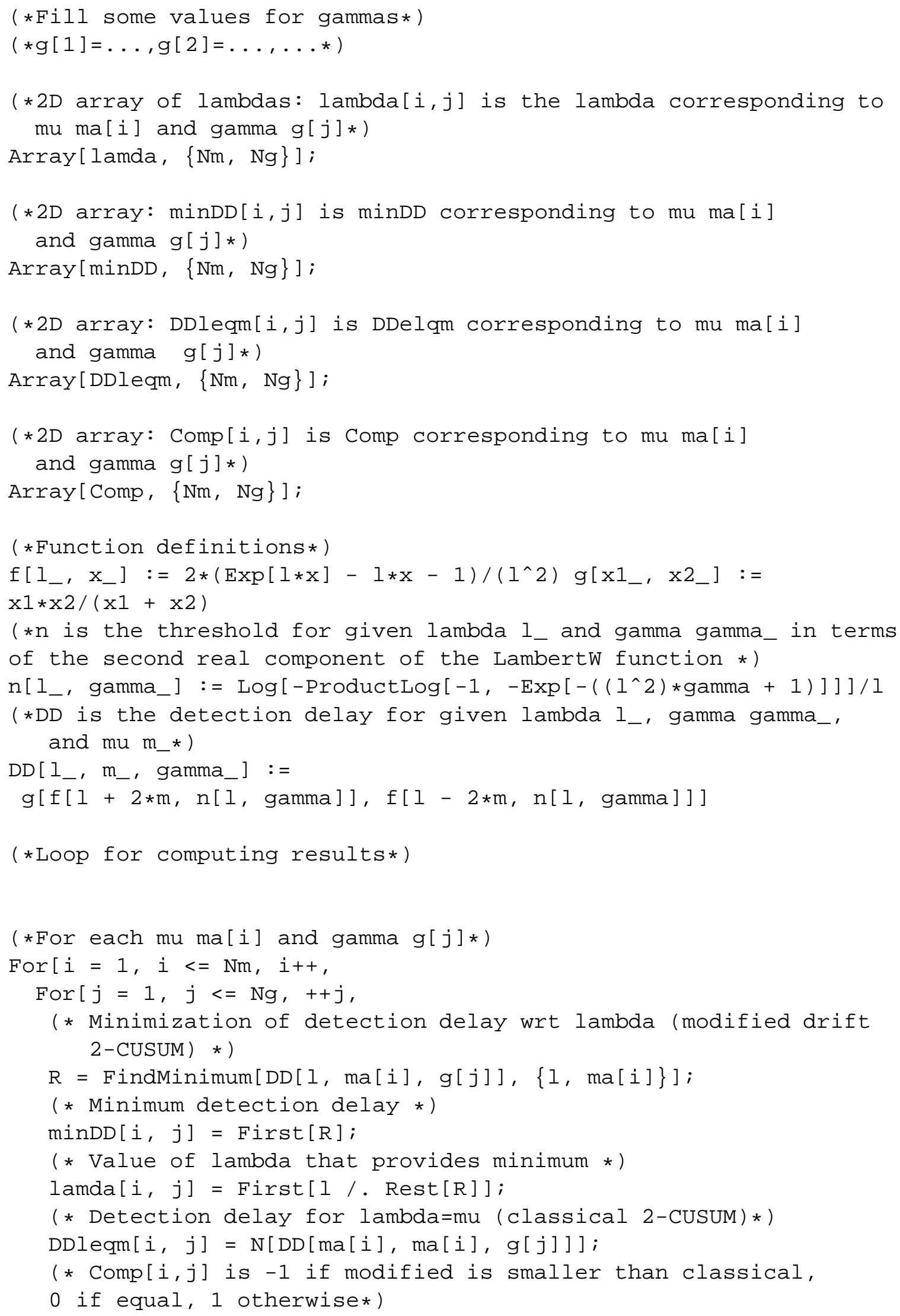




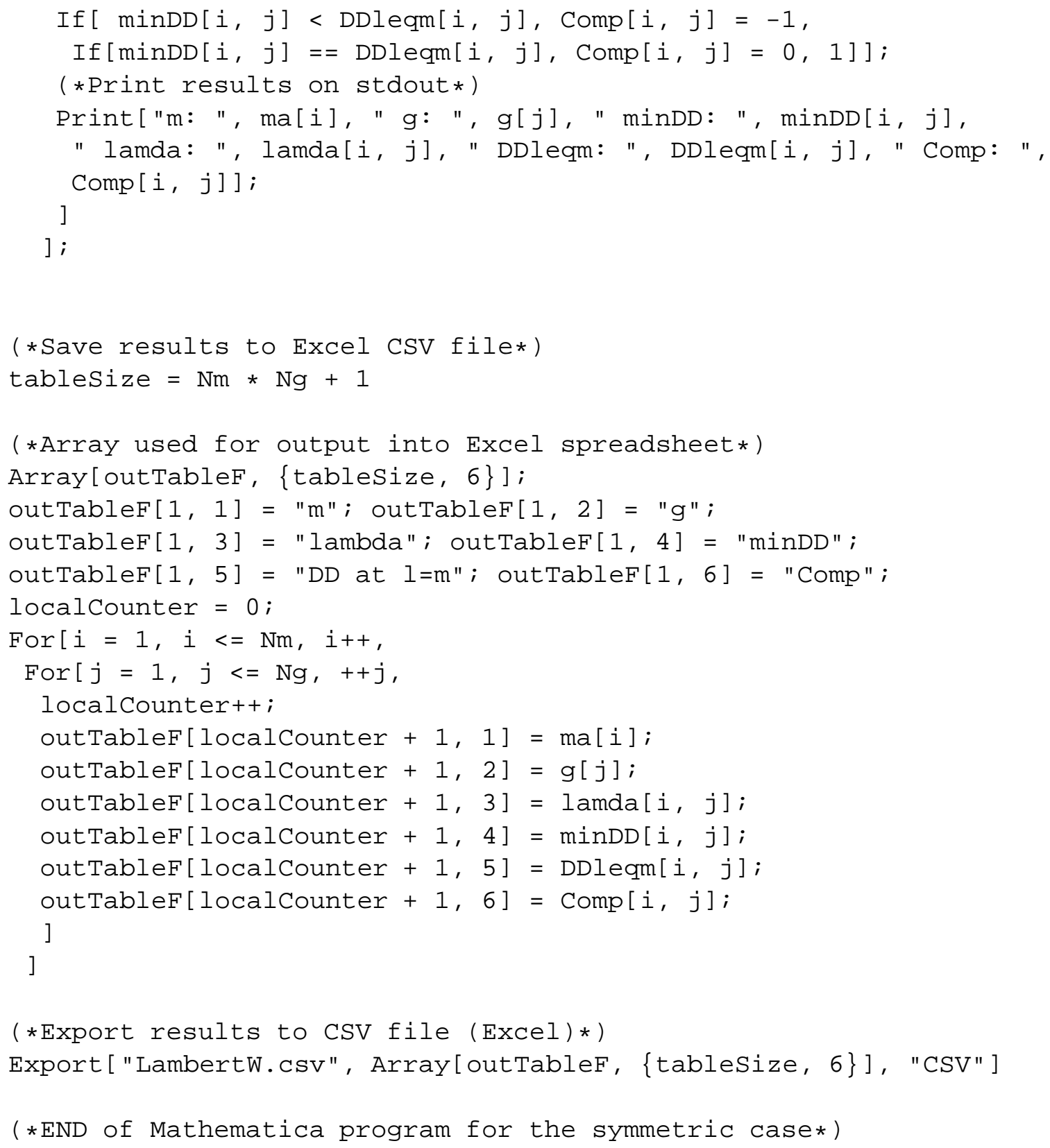

\section{APPENDIX B: NON-SYMMETRIC CASE PROGRAM}

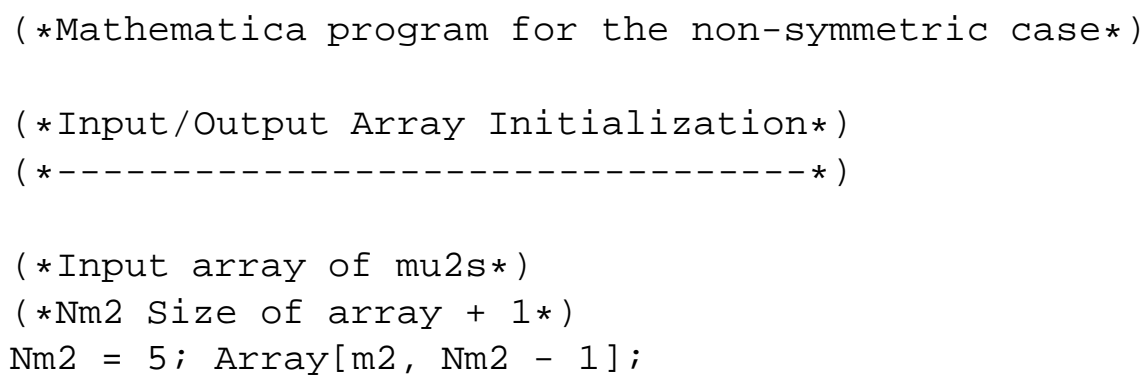




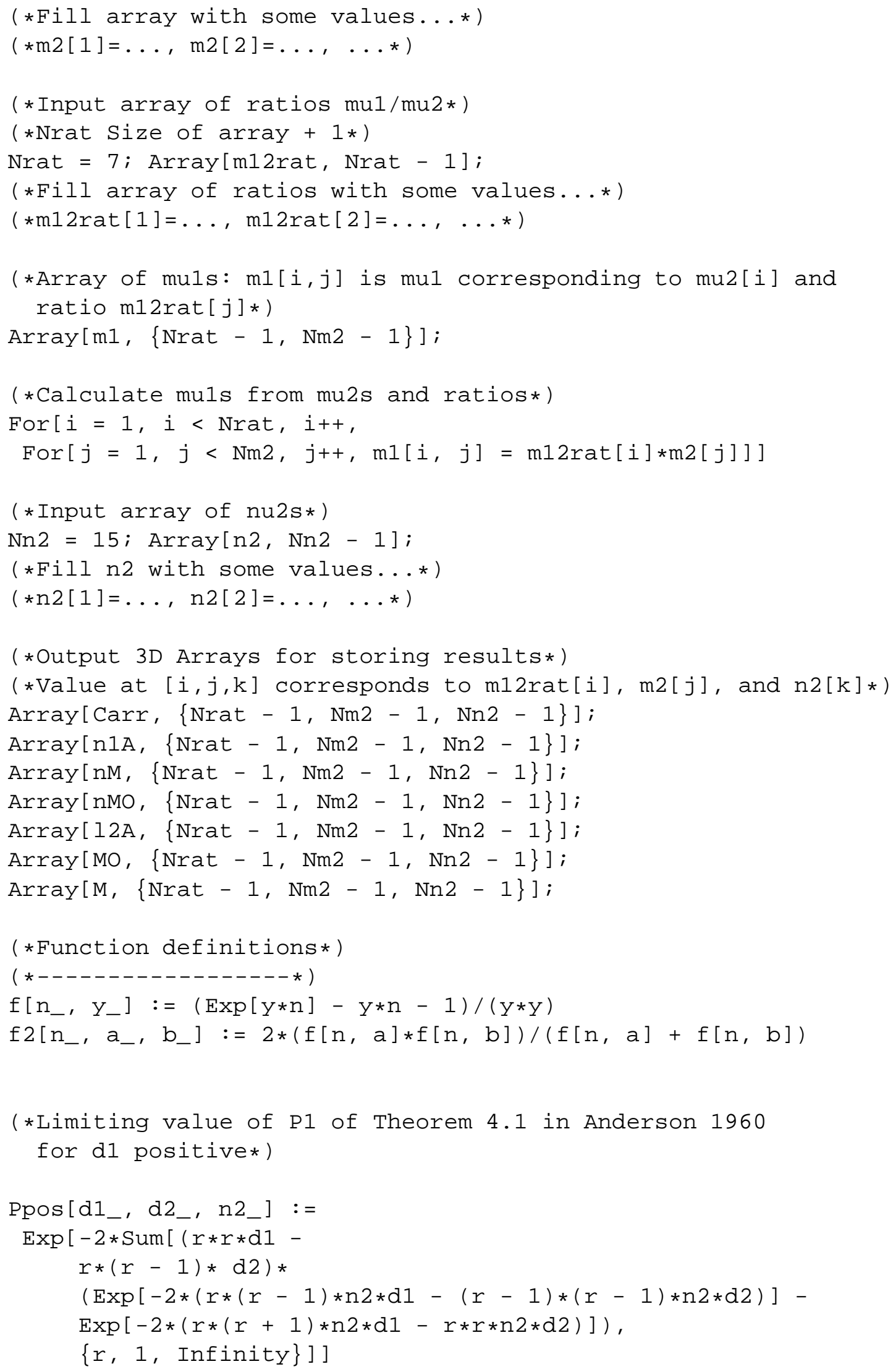




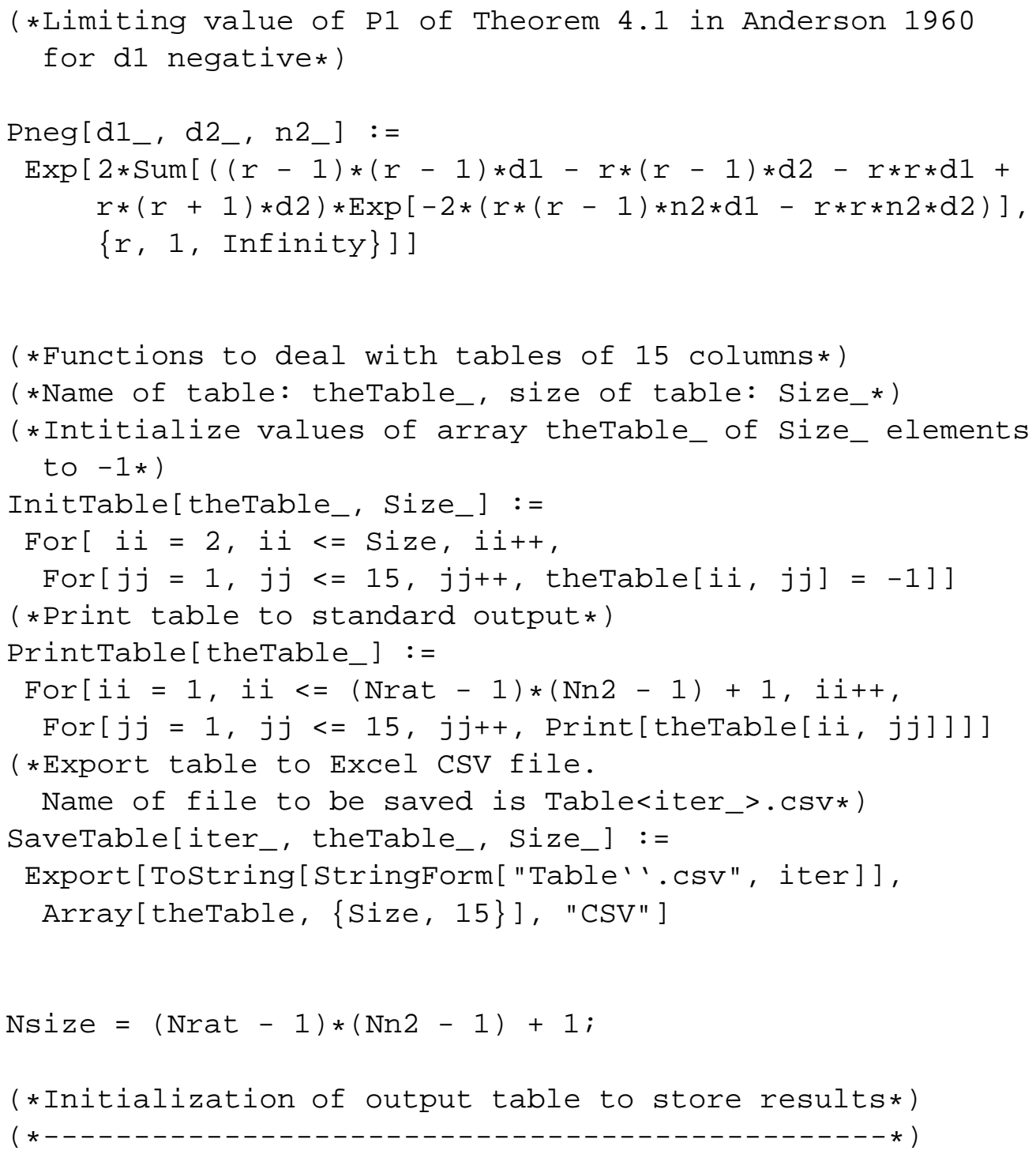

Array [outTable2, \{Nsize, 15\}]; outTable2[1, 1] = "v1"; outTable2[1, 2] = "v2"; outTable2[1, 3] = "m1"; outTable2[1, 4] = "m2"; outTable2 $[1,5]=$ "v1/v2"; outTable $2[1,6]=$ "m1/m2"; outTable2 $[1,7]=$ "nM"; outTable2 $[1,8]=$ "nMO"; outTable2 $[1,9]=" 12 "$; outTable $2[1,10]=$ "g"; outTable2 $[1,11]=$ "C"; outTable2 $[1,12]=$ "M"; outTable $[1,13]=$ "MO"; outTable2 $[1,14]=$ "C $>\mathrm{M}$ ?" ; outTable2 $[1,15]=$ "C>MO ?"; InitTable [outTable2, Nsize];

(*Loop over input values, compute results, and save*)

( $\star-------------------------------------------------\star$ ) 


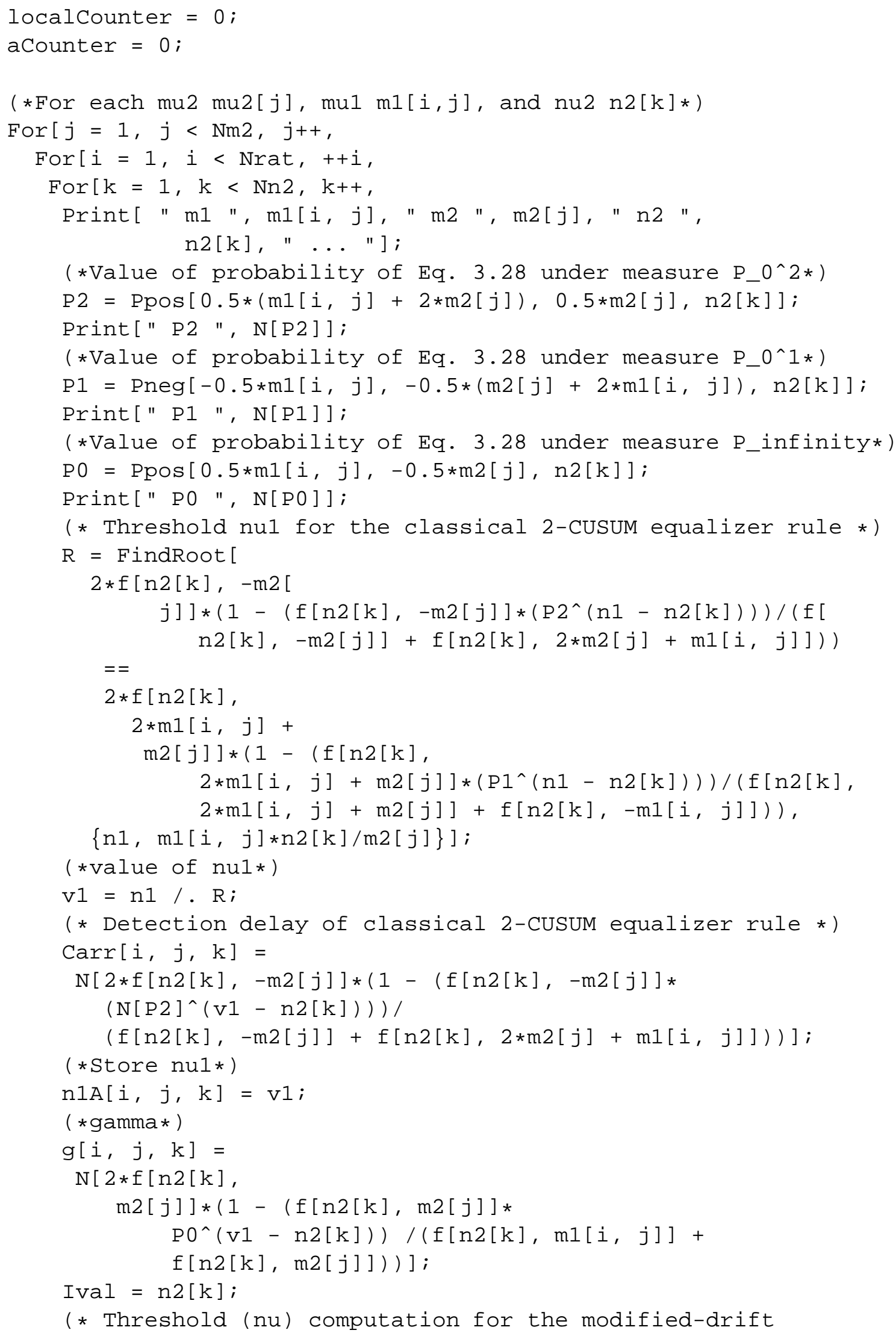




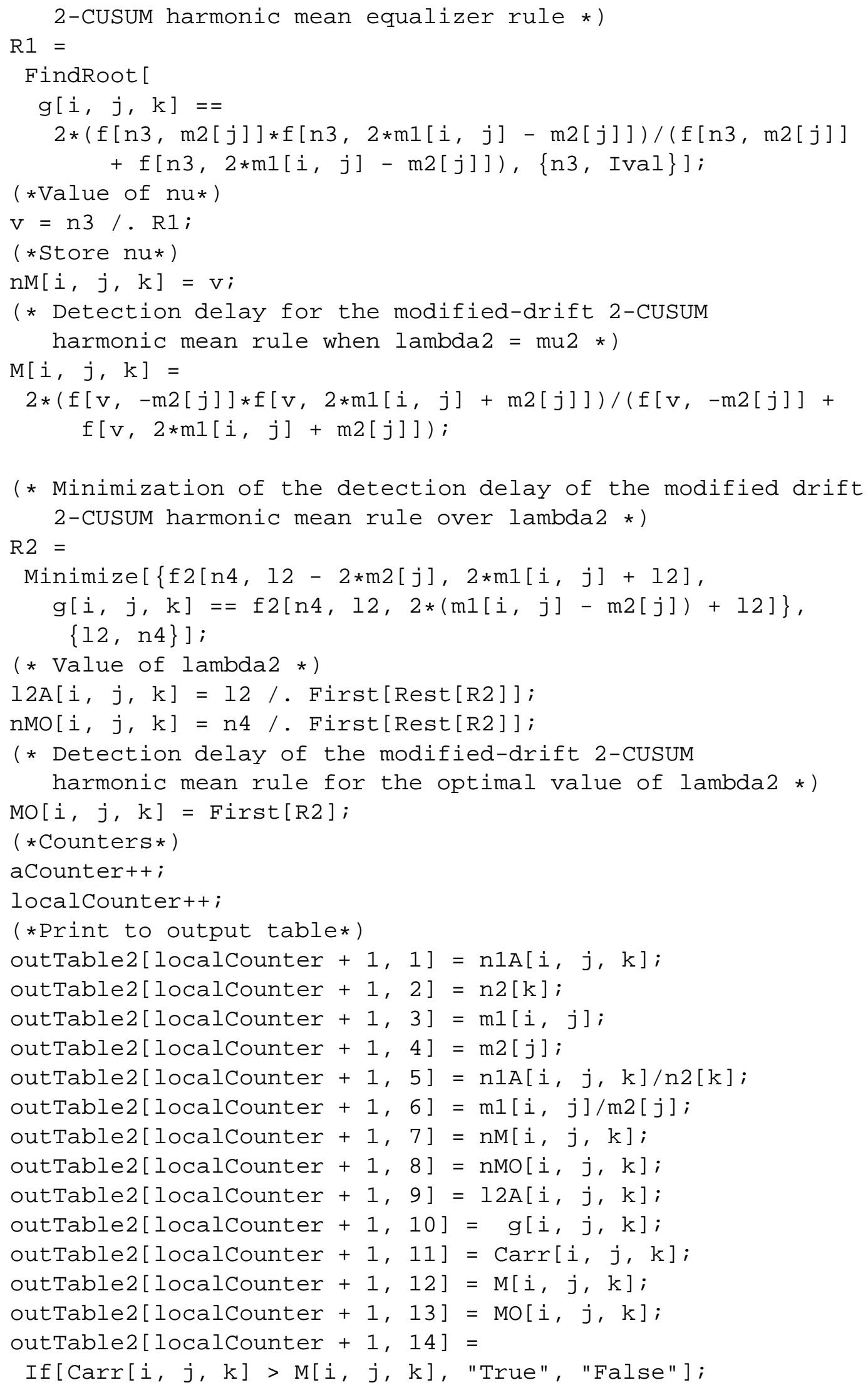




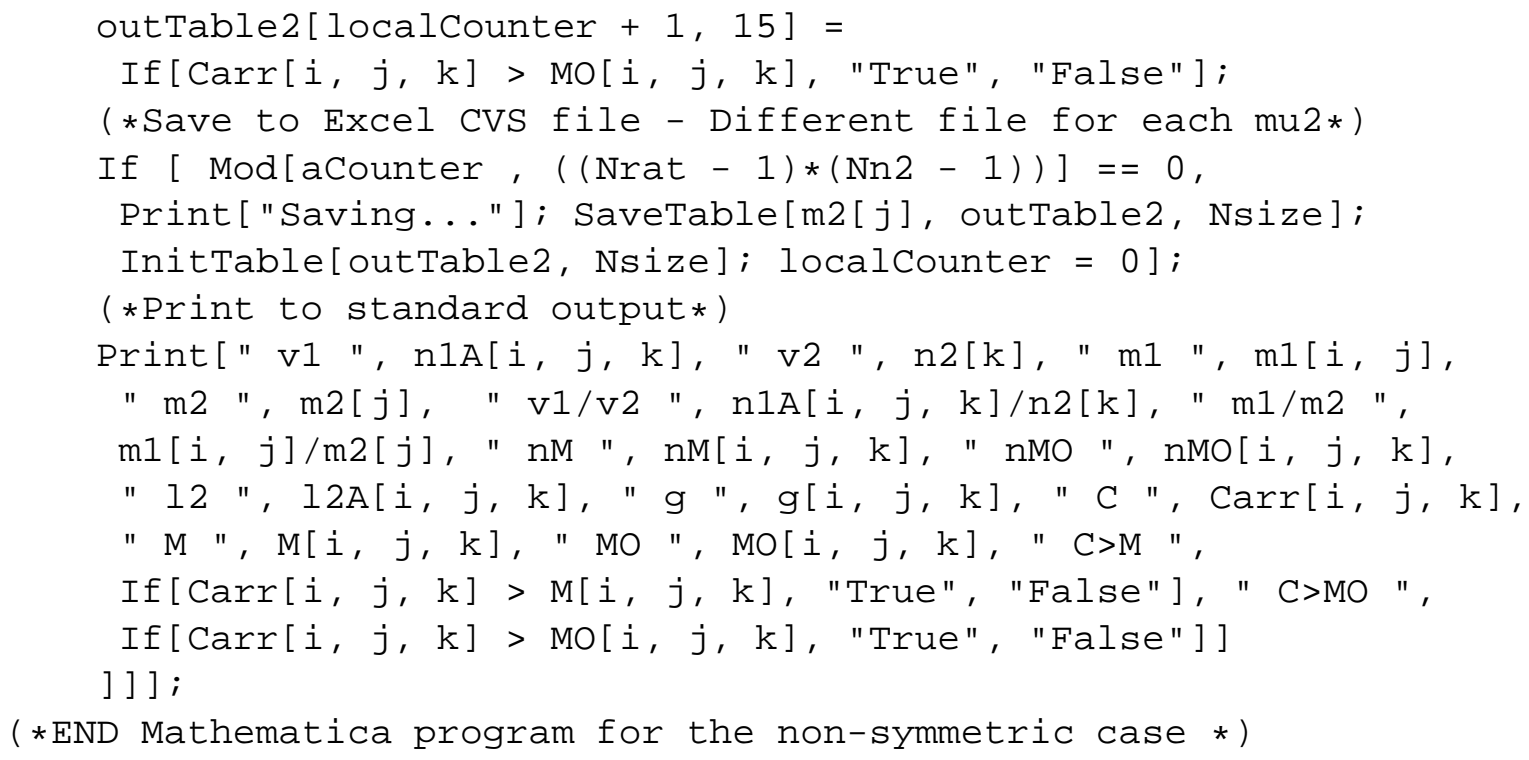

\section{ACKNOWLEDGMENTS}

The authors thank the Editor, an Associate Editor, and the anonymous referees for their helpful suggestions, which have improved the presentation of the article. The authors are also grateful to Dr. George V. Moustakides for introducing the first author to the problem of two-sided detection and to Dr. Peter Carr for introducing the first author to the Lambert W function. The authors are finally grateful to Dr. Mark Brown for the conditioning tool used in (3.17).

\section{REFERENCES}

Anderson, T. W. (1960). A Modification of the Sequential Probability Ratio Test to Reduce the Sample Size, Annals of Mathematical Statistics 31: 165-197.

Anderson, E., Bock, D., and Frisén, M. (2004). Detection of Turning Points in Business Cycles, Journal of Business Cycle Measurement and Analysis 1: 93-108.

Basseville, M. and Nikiforov, I. (1993). Detection of Abrupt Changes: Theory and Applications, New Jersey: Prentice-Hall.

Beibel, M. (1996). A Note on Ritov's Bayes Approach to the Minimax Property of the CUSUM Procedure, Annals of Statistics 24: 1804-1812.

Corless, M., Gonnet, G. H., Hare, D. E. G., Jeffrey, D. J., and Knuth, D. E. (1996). On the Lambert W Function, Advances in Computational Mathematics 5: 329-359.

Doerschuk, P. C., Tenney, R. R., and Willsky, A. S. (1986). Estimation-Based Approaches to Rhythm Analysis in Electrocardiograms, Lecture Notes in Control and Information Science, New York: Springer-Verlag.

Dragalin, V. P. (1997). The Design and Analysis of 2-CUSUM Procedure, Communications Statistics-Simulation and Computation 26: 67-81. 
Hadjiliadis, O. (2005). Optimality of the 2-CUSUM Drift Equalizer Rules Among the Harmonic Mean 2-CUSUM Rule Class for Detecting Two-Sided Alternatives in the Brownian Motion Model, Journal of Applied Probability 42: 1183-1193.

Hadjiliadis, O. and Moustakides, G. V. (2006). Optimal and Asymptotically Optimal CUSUM Rules for Change Detection in the Brownian Motion Model with Multiple Alternatives, Theory or Probability and Applications 50: 131-144.

Hadjiliadis, O. and Poor, H. V. (2008). On the Best 2-CUSUM Stopping Rule for Quickest Detection of Two-Sided Alternatives in a Brownian Motion Model, Theory of Probability and Applications 53.

Hadjiliadis, O. and Vecer, J. (2006). Drawdowns Preceding Rallies in a Brownian Motion Model, Journal of Quantitative Finance 6: 403-409.

Khan, R. A. (1981). A note on Page's two-sided Cumulative Sum Procedure, Biometrika 68: 717-719.

Khan, R. A. (1985). A Stopped Wiener Process Formula with Two Barriers, Sankhya: The Indian Journal of Statistics 47: 285-290.

Khan, R. A. (2007). Distributional Properties of CUSUM Stopping Times, Journal of Sequential Analysis, submitted.

Moustakides, G. V. (1986). Optimal Stopping Times for Detecting Changes in Distributions, Annals of Statistics 14: 1379-1387.

Poor, H. V. and Hadjiliadis, O. (2008). Quickest Detection, Cambridge, UK: Cambridge University Press.

Shiryaev, A. N. (1996). Minimax Optimality of the Method of Cumulative Sums (CUSUM) in the Case of Continuous Time, Russian Math. Surveys 51: 750-751.

Siegmund, D. (1985). Sequential Analysis, New York: Springer-Verlag.

Tartakovsky, A. (1994). Asymptotically Minimax Multi-Alternative Sequential Rule for Disorder Detection, Proceedings Steklov Mathematical Institute 202: 229-236.

Taylor, H. M. (1975) A Stopped Brownian Motion Formula, Annals of Probability 3: 234246.

Willsky, A. S., Chow, E., Gershwin, S. B., Greene, C. S., Houpt, P. D., and Kurkjian, A. L. (1980). Dynamic Model-Based Techniques for the Detection of Incidents on Freeways, IEEE Transactions on Automatic Control 25: 347-359.

Yashchin, E. (1985). On a Unified Approach to the Analysis of Two-Sided Cumulative Sum Control Schemes with Headstarts, Advances in Applied Probability 17: 562593. 